\title{
Parametric optimization of steel floor system cost using Evolver
}

\author{
B. S. Platt \& P. V. Mtenga \\ Department of Civil and Environmental Engineering, Florida A\&M \\ University, Florida State University College of Engineering, USA
}

\begin{abstract}
This paper examines the application of Evolver, a genetic algorithm (GA) solving program, in a three-parameter optimization of a steel truss floor system with a concrete slab floor deck. The floor system is comprised of truss girders supporting beams running in a direction perpendicular to the truss girders with a composite floor deck along the top. Using Evolver, three parameters are optimized for two truss girder topologies in order to find the least cost floor system. The weight of the structural members is correlated to the expenses of material, labor, equipment, overhead, and profit required for the construction of the floor through information given by Means Building Construction Cost Data and interviews with steel fabricators. This procedure may be modified to optimize the cost of any floor area that may use different truss girder topologies, beam sections, and connections.

Parametric optimization is defined in this paper as the combination of configuration, size, and topology optimization of a truss girder, the size optimization of beams, and the optimization of the spacings of both beams and truss girders in the system.
\end{abstract}

Keywords: parametric optimization, cost, steel, truss, topology, configuration, size, Evolver, genetic algorithms, discrete.

\section{Introduction}

A recurrent problem that structural engineers face in building design is the absence of a well-defined method for finding the optimal geometry of floor framing members to minimize cost. The intent of this paper is to introduce a 
method of obtaining the most cost efficient floor system in a large commercial or industrial building through optimizing the geometry of the framing members.

Evolver, a genetic algorithm optimization software program, was chosen for the analysis since it is easy to operate with a brief background of GA and can quickly find improved solutions to the problem. Evolver is used to find the optimal geometry of the framing members that corresponds to the smallest total cost of the steel frame. The proposed method provides engineers a quick and reliable cost estimate for optimized framing parameters where expenses for material, framing connections, labor, shipping, overhead, and profit are a function of the weight of the steel members.

\section{Problem definition}

Two truss topologies, Pratt and Howe, are considered for the truss girder in this study. The beams and truss girders are equally spaced over the floor area with the same size beams and trusses used throughout. The spacing of the beams controls the panel layout of the trusses since it is preferred to place beams at panel points McCormac [1]. A composite metal deck spans across the floor area. Figure 1 illustrates the floor framing system discussed.

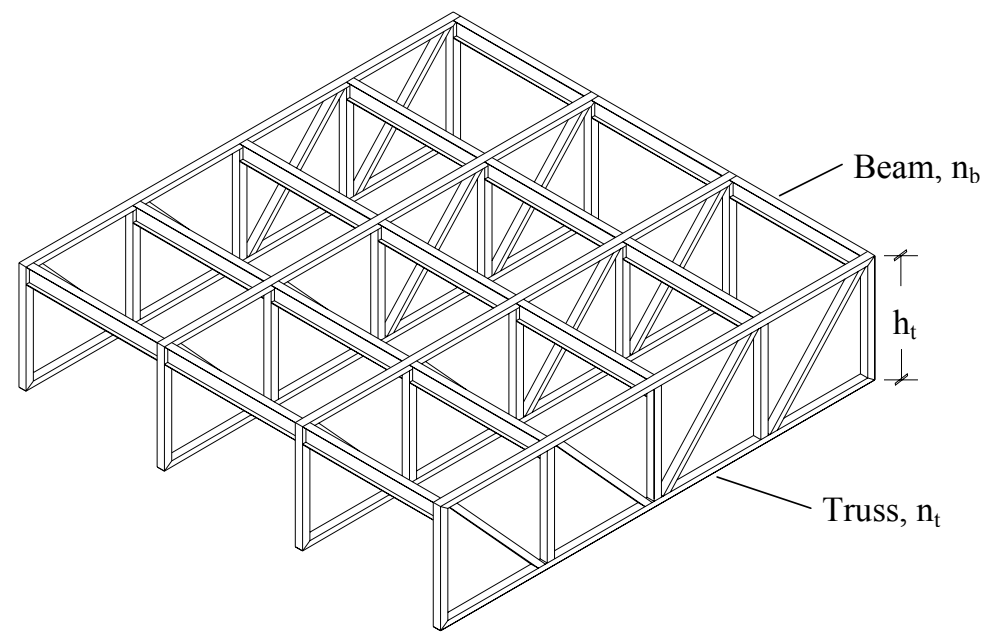

Figure 1: Steel framing system.

\subsection{Methodology}

\subsubsection{Objective function}

The objective function of this study, cost $C$, is to be minimized. A cost coefficient for each beam $c_{b}$ is multiplied by the weight of each beam $w_{b}$, and a separate cost coefficient for trusses $c_{t}$ is multiplied by the weight of each truss $w_{t}$ as shown in eqn (1):

$$
\min (C)=\sum c_{b} \cdot w_{b}+\sum c_{t} \cdot w_{t}
$$


The smallest cost solution for each truss topology considered is compared to one another and the least cost solution is chosen as the optimal configuration.

\subsubsection{Unknowns}

The design parameters of the floor area include the number of truss girders $n_{t}$, height of truss girders $h_{t}$, and number of beams $n_{b}$ for a uniform load.

\subsubsection{Constraints}

The objective function is subject to a set of design and behavioral constraints on the optimization problem, which have been deliberately chosen by limits set on the basis of what is practical in the engineering environment. Design constraints define the boundaries of the design variables. In order to maintain symmetry of the trusses, beams are chosen in odd numbers. The minimum number of beams is three, which produces the minimum number of panels in a Pratt or Howe truss. The maximum number of beams has been chosen through trial and error as 19 . Evolver works more efficiently if the search space is reduced. Similarly, through trial and error, the number of trusses was chosen to be between three and 19. A shallower truss than $3 \mathrm{ft}$ did not seem reasonable since deeper trusses usually require less material. A maximum truss height of $15 \mathrm{ft}$. has been chosen to limit transportation difficulties under roadway bridges. The truss height is varied in whole feet. Also, for ease of welding, the branch member orientations are maintained greater than 30 degrees (AISC Spec. for Steel HSS, Sect. 9.4) [2]. Behavioral constraints include stress, strain, and deflection limitations.

\section{Excel program setup}

An Excel program was developed for this study, comprising four individual tables. These consist of the main design and analysis table where the design parameters are listed and the majority of calculations are done, the beam selection table which is a list of section sizes available for the beams, and the compression member selection table and tension member selection table where a list of sections are provided for each truss member. With the application of Evolver, an add-in for Excel, the floor system cost can be optimized. The Excel program automatically performs a structural analysis for any floor framing geometry, and a total cost is immediately given for the floor system. Different shapes have been assigned to the members to exemplify how various sections can be integrated into the floor system.

\subsection{Main design and analysis table}

Here, the user may enter the dimensions of the floor system, $x_{b}$ and $x_{t}$, corresponding to the directions in which the beams and trusses span. Also, the dead and live loads are entered. Evolver selects the number of beams, number of trusses, and truss height. This is explained later in the paper. The following details the information that must be input into Excel for the program to perform the structural analysis. 


\subsubsection{Initial constraints}

The spacings of the beams and trusses are respectively given by:

$$
s_{b}=\frac{x_{t}}{n_{b}-1}, s_{t}=\frac{x_{b}}{n_{t}-1} .
$$

For ease of welding the diagonal truss members, an angle check is performed:

$$
\max \left[\frac{\sqrt{3}}{3}-\frac{h_{t}}{s_{b}}, \text { if }\left(\frac{h_{t}}{s_{b}}-\sqrt{3}>0, \frac{h_{t}}{s_{b}}-\sqrt{3}, 0\right)\right]
$$

where eqn (4) must equal zero. The thickness of the slab is found by:

$$
t=i f\left[s_{b} \leq 9 . \overline{3} f t, 4 i n, 4 i n+\text { ceiling }\left(\frac{s_{b}-9 . \overline{3} f t}{2 . \overline{3} f t}\right) \text { in }\right] .
$$

\subsubsection{Beams}

W-shaped sections have been selected for the beams of the floor system. The sections are assumed to be compact since minimal weight is desired. The nominal flexural strength is determined by:

$$
M_{n}=\frac{M_{u}}{\phi_{b}}
$$

where $M_{u}$ is the moment due to factored loads and $\phi_{b}$ is the resistance factor for flexure. This is equal to the plastic bending moment $M_{p}$ since the compression flange of the beam is continuously supported by the slab. The required plastic section modulus is found by:

$$
Z_{x}=\frac{M_{p}}{F_{y}}
$$

where $F_{y}$ is the minimum yield stress of the steel (AISC, Sect. F1.1).

The required moment of inertia of the beams is established as:

$$
I_{x, r}=\max \left(\frac{5 w \cdot s_{t}^{4}}{384 E \cdot \Delta_{T L}}, \frac{5 w_{L L} \cdot s_{t}^{4}}{384 E \cdot \Delta_{L L}}\right)
$$

where $E$ is the modulus of elasticity of the steel and $\Delta_{T L}$ and $\Delta_{L L}$ are the allowable deflections for total load and live load, respectively.

\subsubsection{Truss members}

A pattern for automatically solving the forces in the truss members has been established. Starting from the ends of the truss and moving toward the center, the truss member forces are solved. In a Howe truss, the center vertical member is a zero-force member, tending toward tension, $T_{l}$. The other zero-force members are located at the top chord segments furthest to the truss ends, tending toward compression, $C_{2}$. Each panel of the truss girder consists of four members. For three beams, only one panel is designed: 


$$
\begin{gathered}
C_{3}=P_{e}, \\
C_{4}=\frac{\left(V-C_{3}\right) \sqrt{s_{b}^{2}+h_{t}^{2}}}{h_{t}}, \\
T_{5}=\frac{C_{4} \cdot s_{b}}{\sqrt{s_{b}^{2}+h_{t}^{2}}} .
\end{gathered}
$$

For more than three beams, a counting factor $i$ is applied to include each additional panel's set of four members. Two double-panels are designed for 5 beams, three double-panels for 7 beams, and so on. For each additional double-panel beyond the initial 2-panel truss:

$$
\begin{gathered}
i=1,2,3, \ldots, n_{p}, \\
C_{2+4 i}=i f\left(n_{b} \leq 3+2(i-1), 0, T_{5+4(i-1)}\right), \\
T_{3+4 i}=i f\left[n_{b} \leq 3+2(i-1), 0, \frac{C_{4 i} \cdot h_{t}}{\sqrt{s_{b}^{2}+h_{t}^{2}}}-P_{i}\right], \\
C_{4+4 i}=i f\left[n_{b} \leq 3+2(i-1), 0, \frac{T_{3+4 i} \sqrt{s_{b}^{2}+h_{t}^{2}}}{h_{t}}\right], \\
T_{5+4 i}=i f\left[n_{b} \leq 3+2(i-1), 0, T_{1+4 i}+\frac{C_{4+4 i} \cdot s_{b}}{\sqrt{s_{b}^{2}+h_{t}^{2}}}\right] .
\end{gathered}
$$

For clarity, Figure 2 labels each truss member and the number of additional double-panels $n_{p}$. The required radius of gyration about the $y$-axis for the zeroforce members are as follows:

$$
r_{y_{1}}=\frac{K_{b} \cdot h_{t}}{300}, r_{y_{2}}=\frac{K_{c} \cdot s_{b}}{200}
$$

where $K_{b}$ and $K_{c}$ are the effective length factors for branch and chord members respectively (AISC Spec. for Steel HSS, Sect. 2.3, 4.1.1 (a)) [2].

For the other members, the required effective lengths are:

$$
\begin{gathered}
K L_{3}=\text { ceiling }\left(K_{b} \cdot h_{t}, 1 f t\right), \\
K L_{4}=\text { ceiling }\left(K_{b} \sqrt{s_{b}^{2}+h_{t}^{2}}, 1 f t\right), \\
L_{5}=\text { ceiling }\left(s_{b}, 1 f t\right), \\
K L_{2+4 i}=i f\left[C_{2+4 i}=0,0, \text { ceiling }\left(K_{c} \cdot s_{b}, 1 f t\right)\right],
\end{gathered}
$$


124 Computer Aided Optimum Design in Engineering X

$$
\begin{aligned}
L_{3+4 i}= & \text { if }\left[T_{3+4 i}=0,0, \text { ceiling }\left(h_{t}, 1 f t\right)\right], \\
K L_{4+4 i}= & \text { if }\left(C_{4+4 i}=0,0, \text { ceiling }\left(K_{b} \sqrt{s_{b}^{2}+h_{t}^{2}}, 1 f t\right)\right), \\
L_{5+4 i} & =\text { if }\left[T_{5+4 i}=0,0, \operatorname{ceiling}\left(s_{b}, 1 f t\right)\right] .
\end{aligned}
$$

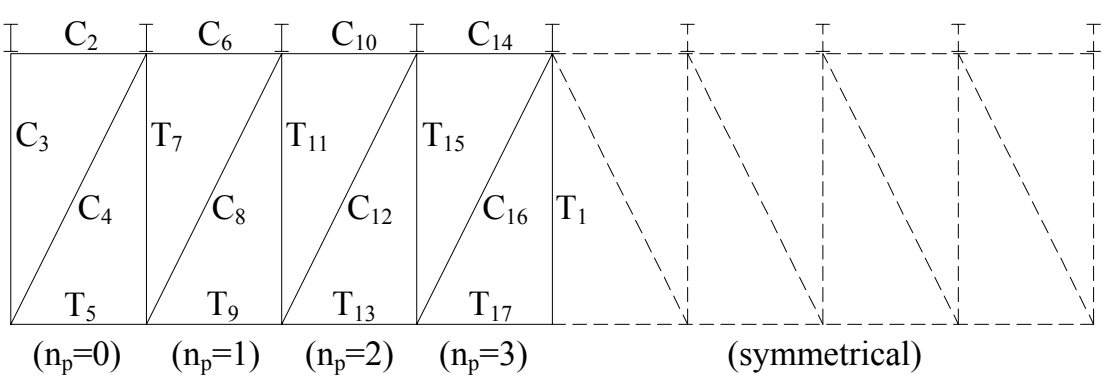

Figure 2: $\quad$ Truss layout.

A cross-sectional area is assigned to each member from either the compression or tension member selection table. The largest force of all chord segments is used to size the entire chord. When finding the weight per foot of each member:

$$
\begin{aligned}
& w_{1}=3.4 \frac{p s i}{f t} A_{g_{1}}, \\
& w_{2}=3.4 \frac{p s i}{f t} \max \left(A_{g_{2}}, A_{g_{6}}, A_{g_{10}}, \ldots, A_{g_{2+4 n_{p}}}\right) \text {, } \\
& w_{3}=3.4 \frac{p s i}{f t} A_{g_{3}}, w_{4}=3.4 \frac{p s i}{f t} A_{g_{4}}, \\
& w_{5}=3.4 \frac{p s i}{f t} \max \left(A_{g_{5}}, A_{g_{9}}, A_{g_{13}}, \ldots, A_{g_{5+4 n_{p}}}\right) \text {, } \\
& w_{2+4 i}=i f\left[n_{b} \leq 3+2(i-1), 0,3.4 \frac{p s i}{f t} \max \left(A_{g_{2}}, A_{g_{6}}, A_{g_{10}}, \ldots, A_{g_{2+4 n_{p}}}\right)\right] \text {, } \\
& w_{3+4 i}=3.4 \frac{p s i}{f t} A_{g_{3+4 i}}, \\
& w_{4+4 i}=3.4 \frac{p s i}{f t} A_{g_{4+4 i}},
\end{aligned}
$$




$$
w_{5+4 i}=i f\left[n_{b} \leq 3+2(i-1), 0,3.4 \frac{p s i}{f t} \max \left(A_{g_{5}}, A_{g_{9}}, A_{g_{13}}, \ldots, A_{g_{5+4 n_{p}}}\right)\right] \text {. }
$$

Total and live load deflections of the truss are checked through the method of virtual work. If deflection is not satisfied for a particular solution, the chord members of the truss are enlarged to the next smallest section and deflection is again checked. The process is repeated until deflection is satisfied.

\subsubsection{Total cost}

The total cost of the floor system is written in terms of the total costs of the beams and trusses:

$$
C=c_{b} W_{b}+c_{t} W_{t}
$$

where $W_{b}$ and $W_{t}$ are the total weights of the beams and trusses.

\subsection{Member selection tables}

\subsubsection{Beam selection table}

$A_{g}, I_{x}$, and $Z_{x}$ are input into the Excel beam selection table for standard AISC sections. For each section, two checks for $I_{x}$ and $Z_{x}$ are performed together in a separate column. If its $I_{x}$ is greater than or equal to the required $I_{x}$, then its area is selected. Otherwise, a default area of $9,999 \mathrm{in}^{2}$ is selected. Then, if its $Z_{x}$ is greater than or equal to the required $Z_{x}$, then its area is selected. Otherwise, the default area of 9,999 in is selected. The maximum area of the two cases is displayed for each section. The smallest area of these represents the least-weight member that satisfies the criteria. The process is repeated and a new section is found if the previous section was insufficient for the addition of its self-weight.

\subsubsection{Tension member selection table}

The radius of gyration $r_{y}$, gross area $A_{g}$, and tension design strength $\phi_{t} P_{n}$ are input into the Excel tension member selection table for standard AISC sections. For the zero-force member, each section is checked to see if its $r_{y}$ is at least the value of the required $r_{y}$. If this is satisfied, its area is selected. Otherwise, a default area of 9,999 in ${ }^{2}$ is selected. After slenderness limitations are verified, each section's design strength is checked to ensure that it equals or exceeds the acting tension force. If either criterion is not satisfied, the section is assigned an area of 9,999 in in a separate row. Otherwise, the section's cross-sectional area is displayed in the row. The smallest area displayed for all of the cross-sections corresponds to the least-weight section that satisfies the criteria. Every crosssectional area selected for the tension members in the tension member selection table is linked to the main design and analysis table to include with the total weight of the floor system. 


\subsubsection{Compression member selection table}

The Excel compression member selection table was created in a similar format as AISC tables, where $\phi_{c} P_{n}$ is listed for a range of effective lengths for each section. The process for selecting cross-sectional areas is similar to that of the tension member selection table.

\section{Cost analysis}

Total cost includes expenses for material, preparation of members, shop drawings, labor, overhead and profit, transportation, and installation.

\subsection{RSMeans}

The framing system is assumed to be part of a new commercial or industrial building. For this project size, the Means Heavy Construction Cost Data book is referenced in order to develop an accurate and current construction cost estimate for the framing of the floor system. RSMeans [3] does not have an applicable listing for the HSS trusses used in this study.

The costs for the W-section beams are based on data from RSMeans. Costs for approximately $30 \%$ of the members listed in the AISC manual are given in RSMeans. An average cost per weight of steel is taken for specific ranges for which information is listed, and member weights calculated within those ranges are assigned the corresponding costs.

\subsection{Steel fabricators}

Estimates from five steel fabricators, [4-8] in the southern United States were used in this study. It is recommended that the designer compare prices with several fabricators in their area to save money in construction costs. While some of the steel fabricators interviewed were not at liberty to disclose specific details of their cost estimations, they were willing to provide a general breakdown of the total cost of materials and labor for a typical beam and truss used in the system. Beam estimates given by the steel fabricators were similar to those listed in the RSMeans catalogue.

Truss cost estimations given by the steel fabricators are based on fully shop assembled trusses. Two trusses were given to each of the fabricators to develop cost estimates. The first was a truss in an 80'x80' floor area, and the other was in a 40' $\times 40$ ' floor area. The member sections were chosen based on optimized weight. In general, they tended to find the cost of each of these items in terms of the total weight of each truss. Through an analysis of the estimates provided, the cost of trusses has been assumed as $\$ 6.00 / 1 b$ for calculations. The cost per pound for smaller trusses increases because the cost of small sections is more per pound than for larger sections. Cheaper costs per pound for heavier sections are compromised by more expensive shipping and handling costs and costs for splicing members if necessary. 


\section{Genetic algorithms}

A genetic algorithm starts with an initial population of " $n$ " individual chromosomes corresponding to different solutions to the problem. The makeup of each chromosome for this study is composed of three segments: $n_{b}, n_{t}$, and $h_{t}$. Mutation and crossover operational adjustments are carried out after several generations without improvement of the objective function.

\section{Evolver}

Evolver is an add-in for Microsoft Excel that finds better solutions to optimization problems by adjusting variables through genetic algorithms. Working with the writer's Excel cost program, Evolver rapidly adjusts $n_{b}, n_{t}$, and $h_{t}$ to progress to the most optimal solution. Evolver is easy to operate, using a similar format as the Excel Solver add-in. The program allows the user to find the minimum value in Excel for the cost by automatically adjusting the variables. In order to avoid interpolation and to use only discrete variables, integer values are selected for the parameters Palisade [9].

The given range for each adjustable cell is a hard constraint that is strictly enforced. Although Evolver will not converge to an infeasible solution, it evaluates infeasible solutions in order to search for feasible areas Palisade [9]. Additional hard constraints placed on the solution include the selection of an odd number of beams, the 30 degree minimum branch member orientation from both horizontal and vertical truss members, and the total load and live load truss deflection limitations.

An example of a typical graph produced by Evolver is shown in Figure 3. The best solution found thus far for each trial is plotted. This particular graph is for a Howe truss in a 40'x40' floor area. The initial solution was about $\$ 52,000$. After a period of time with no change in the best solution (50-100 trials), the crossover and mutation rates were altered.

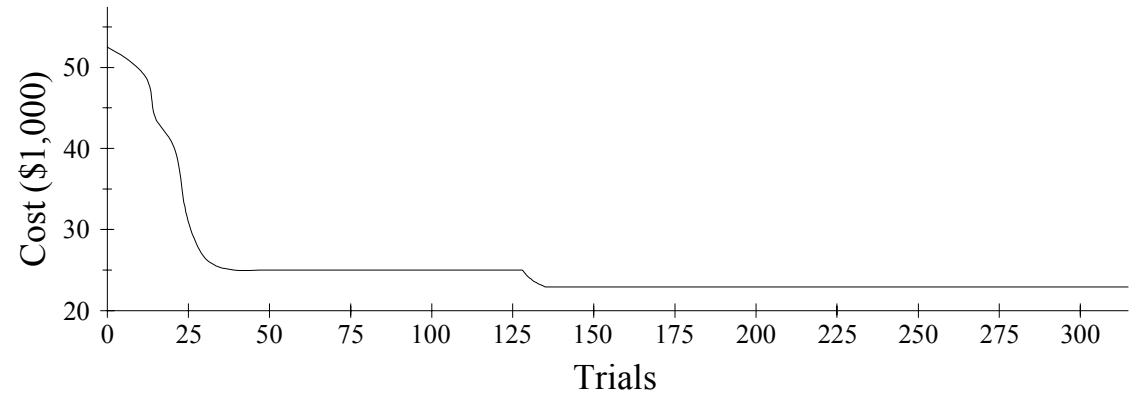

Figure 3: Plot of Evolver's progression. 
After several generations with no change in the most optimal solution, $\$ 23,000$ was taken as the final solution. It is not possible to tell whether a GA has found the global optimum answer to a problem, but it is possible for it to have found a much improved answer. This took approximately three minutes for Evolver to optimize.

\section{Conclusion}

A procedure for automatically choosing the most efficient floor framing for any loading condition or floor area becomes invaluable to an engineer. This cuts out the guesswork of having to calculate different trials by hand and taking time to look up values in tables, or developing structural models in design programs for each floor frame considered. A spreadsheet can be developed that automatically assigns sections to members of any floor system, similar to the one created for this study. Evolver is an optimization tool that can be used with an Excel spreadsheet to minimize the cost of a floor frame. After inputting the parameters, it should only take about five minutes to run the analysis and find a much improved solution. Where cost is determined as a function of the weight of the members, it is not necessary to detail the connections of the system to come up with a total estimate. This technique can be functional to both professionals and students in the fields of engineering and optimization. This is a basic procedure that can be customized for the cost optimization of any size floor or roof area that may use different truss girder topologies, beam sections, and connections.

\section{References}

[1] McCormac, J.C., Structural Analysis, $4^{\text {th }}$ Ed., HarperCollins Publishers: New York, pp. 85, 87, 1984.

[2] American Institute of Steel Construction, Manual of Steel Construction. Load and Resistance Factor Design, $3^{\text {rd }}$ Ed., AISC: Chicago, 2001.

[3] RSMeans, Heavy Construction Cost Data, 20 ${ }^{\text {th }}$ Annual Ed., Reed Construction Data, Inc.: Kingston, MA, 2006.

[4] Davis, D. Personal Communication, 14 April 2006, Project Manager, Bell Steel Company, Pensacola, FL.

[5] Montgomery, J. Personal Communication, 1 February 2006, Manager, Southland Tube, Inc., Montgomery AL.

[6] Tucker, S. Personal Communication, 17 April 2006, Project Manager, Southern Steel Company, LLC, Charlotte, NC.

[7] Turner, H.R. Personal Communication, 10 April 2006, Vice President/ Estimating and Sales, Qualico Steel Company, Inc., Webb, AL.

[8] Williams, B. Personal Communication, 31 January 2006, Manager, Jackson-Cook, LC, Tallahassee, FL.

[9] Palisade Corporation, The Guide to Evolver: The Genetic Algorithm Solver for Microsoft Excel, Palisade Corporation: Newfield, NY, pp. 27, 101, 108, $116,2001$. 